\title{
Profile of isavuconazole and its potential in the treatment of severe invasive fungal infections
}

This article was published in the following Dove Press journal:

Infection and Drug Resistance

22 October 2013

Number of times this article has been viewed

\author{
Diego R Falci ${ }^{1,2}$ \\ Alessandro C Pasqualotto 2,3 \\ 'Hospital Nossa Senhora da \\ Conceição, Porto Alegre, Brazil; \\ 2Universidade Federal de Ciências da \\ Saúde de Porto Alegre, Porto Alegre, \\ Brazil; ${ }^{3}$ Santa Casa de Misericórdia de \\ Porto Alegre, Porto Alegre, Brazil
}

Correspondence: Alessandro C

Pasqualotto

Molecular Biology Laboratory, Santa Casa de Misericordia de Porto Alegre, Avenue Independência 155, $8^{\circ}$ andar, 90035-074,

Porto Alegre, Brazil

Tel +55 5। 9995 ।6I4

Fax +55 51 3213 749।

Email acpasqualotto@hotmail.com

\begin{abstract}
The triazole class of antifungal drugs comprises first-line agents for the treatment of several invasive fungal diseases. Isavuconazole is a novel broad-spectrum triazole agent. Here we summarize its characteristics and compare it with the currently available antifungal agents. Isavuconazole is administered as a prodrug, and it is water soluble. Oral and intravenous formulations are available. Its intravenous formulation does not contain cyclodextrin, which is an advantage over voriconazole, considering the potential for nephrotoxicity of cyclodextrin. As with other azoles, isavuconazole requires a loading dose. Due to its prolonged half-life, a once-a-day regimen is possible. Considering that isavuconazole shares the same mechanism of action with the other triazoles, cross-resistance is an important concern in the class. Tolerability and safety profiles are favorable, and no serious adverse events have been consistently reported. Significant interactions with drugs metabolized by cytochrome P450 are expected to occur, especially with substrates and inducers of the CYP3A4 enzyme. Isavuconazole has in vitro activity against most medically important fungi, including species of Candida, Aspergillus, and Cryptococcus. It has some activity against the agents of mucormycosis. Clinical data regarding isavuconazole remain limited because ongoing trials have not yet been completed or published. Isavuconazole has the potential to become first-line therapy for invasive aspergillosis. It also has the potential for use in the context of antifungal prophylaxis, salvage therapy, or in combination regimens. Results of clinical trials are ultimately expected in order to adequately position isavuconazole in the current antifungal armamentarium.
\end{abstract}

Keywords: isavuconazole, invasive fungal infections, antifungals, triazoles, Aspergillus

\section{Introduction: current scenario of antifungal therapy}

The incidence of opportunistic invasive fungal diseases (IFDs) has increased in the last decades, and a change in the spectrum of fungal pathogens has been observed. Infections due to molds other than Aspergillus and yeasts other than Candida have become increasingly common in this new scenario. ${ }^{1}$ Fortunately, our options to treat patients with deadly fungal infections have evolved from the toxic compounds available in the 1980 s to more effective and safe antifungal drugs in the new millennium. ${ }^{1-3}$ In addition, progress in the diagnosis of IFDs with the use of non-culture-based techniques has substantially improved the prognosis of patients with fungal infections. However, despite the arrival of the age of modern antifungal therapy, some therapeutic problems remain - in particular, the emergence of new pathogenic fungal species, slow microbiological diagnosis, variable drug bioavailability, drug toxicities, lack of either oral or intravenous (IV) drug preparations, significant drug interactions for some agents, and development of antifungal resistance or breakthrough infections. 
The triazole class of antifungal drugs comprises first-line agents for the treatment of IFDs caused by a range of medically important opportunistic fungal pathogens. These agents vary quite markedly, however, in terms of structure-activity relationships, pharmacokinetics, toxicity, pharmacological interactions, and clinical use. ${ }^{1-3}$ Voriconazole has a major role in the treatment of invasive aspergillosis (IA) as well as other mold infections such as fusariosis. Fluconazole is useful in the prophylaxis and treatment of Candida infections, but its indication for patients with candidemia has somewhat been overcome by the arrival of the echinocandins, mainly because of their wider spectrum of action and safety profile. Posaconazole use has remained mostly restricted to antifungal prophylaxis, considering the lack of an IV formulation and clinical experience for use in the treatment of IFDs. Itraconazole has the same problems as posaconazole plus an erratic absorption, narrower spectrum of antifungal activity, and many pharmacological interactions. For Candida infections, the echinocandins (caspofungin, anidulafungin, and micafungin) have demonstrated superiority to other classes, due to their excellent safety profile, great fungicidal effect against the genera, and proven efficacy in clinical grounds and trials. The polyenes (amphotericin B deoxycholate and its lipid formulations) - once considered the mainstay of antifungal treatment - have a more limited role now, particularly due to safety concerns. The role of polyenes, however, remains quite important in the treatment of mucormycosis, infections by Cryptococcus, and other emerging yeasts, such as the dimorphic fungi, as well as salvage therapy for several mold and yeast infections.

The aim of this review is to summarize the in vitro, in vivo, and clinical data available on a novel broad-spectrum triazole agent azole, isavuconazole (formerly BAL4815; Basilea Pharmaceutica, Basel, Switzerland, and Astellas Pharma, Tokyo, Japan) reflecting on its potential use in the clinical practice, and to compare it with currently available agents.

\section{What is the chemical structure of isavuconazole and how different is it from the other azoles?}

Isavuconazonium (BAL 8557, formerly RO-0098557) is a prodrug of isavuconazole (BAL 4815). BAL 8557 is water soluble and consists of an N-(3-acetoxypropyl)-Nmethylamino-carboxymethyl group attached to isavuconazole via an ester moiety. Isavuconazole's chemical formula is $\mathrm{C}_{22} \mathrm{H}_{17} \mathrm{~F}_{2} \mathrm{~N}_{5} \mathrm{OS}^{4}$ Isavuconazonium is rapidly cleaved into the active component (isavuconazole) and an inactive prodrug cleavage product (BAL 8728) after oral or IV administration.
The chemical structure of the prodrug, active drug, and cleavage product is illustrated in Figure 1. The conversion is rapid and complete, with very low levels of the cleavage product detectable in the serum after oral or IV administration. Unlike the IV formulations of itraconazole and voriconazole, isavuconazonium does not require the addition of cyclodextrin to facilitate solubility, therefore eliminating concerns for nephrotoxicity due to the sulfobutylether- $\beta$-cyclodextrin component. Unlike posaconazole, which is available only in an oral formulation, BAL 8557 can be administered orally or by IV. The oral formulation is a hard gelatin capsule..$^{2,3,5}$ The current therapeutic regimen is $200 \mathrm{mg}$ every 8 hours (IV or orally) for the first 2 days, followed by $200 \mathrm{mg}$ per day (IV or orally). ${ }^{2}$

The water solubility, and consequently the availability of an IV formulation that does not require cyclodextrin, is a clear advantage of isavuconazole in comparison with other azoles such as voriconazole and posaconazole. The need for a loading dose also occurs with voriconazole, for instance, but the possibility of a once-a-day regimen and the interchangeability of dosing between oral and IV administration can be positive differentials favoring isavuconazole.

\section{Similarities with, and differences from, other azoles: antifungal resistance, safety, toxicity, pharmacokinetic/pharmacodynamic parameters, and drug-drug interactions}

As with other members of the triazole class of antifungal drugs, isavuconazole inhibits cytochrome P450 sterol $14-\alpha$-demethylase (CYP51) involved in the synthesis of ergosterol. This blockade produces methylated sterols (eg, 14- $\alpha$-methylated lanosterol, 4,14-dimethylzymosterol, and 24-methylenedihydrolanosterol) in the fungal membrane, which alter its function, resulting in the accumulation of ergosterol toxic precursors in the fungal cytoplasm. The side arm of the active form provides the wide antifungal spectrum being responsible for orientating the molecule, so that the triazole ring can engage with the heme moiety at the bottom of the binding pocket in the fungal CYP51 protein., ${ }^{2,6}$

The side arm of the isavuconazole molecule will also influence the enhanced activity of this drug against other fungal pathogens that are commonly resistant to the other triazoles (ie, itraconazole, voriconazole, and posaconazole). Cross-resistance among the azoles is still not well understood, but there are relevant studies in progress. An Indian study 


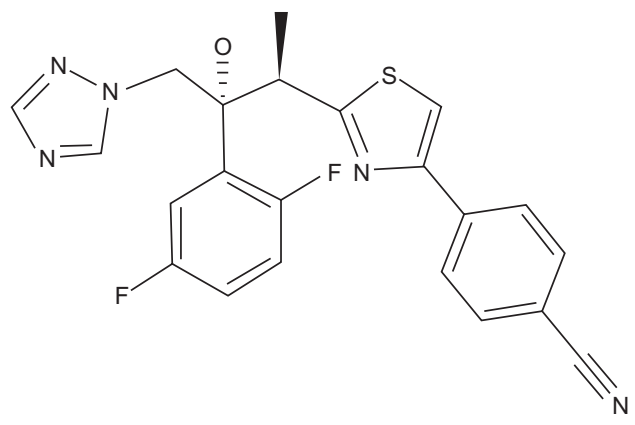

Isavuconazole (active drug BAL 4815)



Cleavage product (BAL 8728)

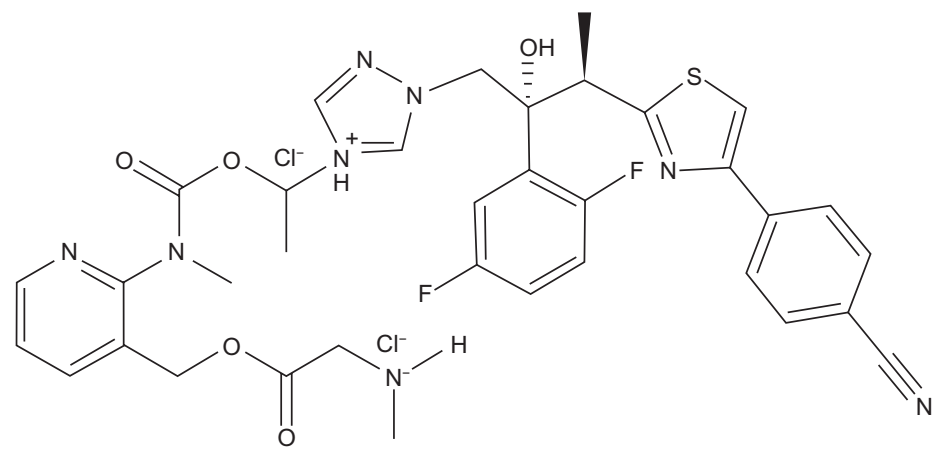

Isavuconazonium (prodrug BAL 8557)

Figure I Chemical structure of the prodrug, active drug, and cleavage product of isavuconazole.

revealed that two Aspergillus fumigatus isolates that were pan-triazole resistant (with itraconazole, voriconazole, and posaconazole minimum inhibitory concentration [MIC] of $>16 \mu \mathrm{g} / \mathrm{mL}, 2 \mu \mathrm{g} / \mathrm{mL}$, and $2 \mu \mathrm{g} / \mathrm{mL}$, respectively) also had elevated isavuconazole MIC $(8 \mu \mathrm{g} / \mathrm{mL}){ }^{7}$ Resistance in these isolates was associated with tandem repeat/L98H mutation. At the moment, is not known whether isavuconazole could be a substrate for efflux pumps in Aspergillus spp. Considering the potential public health catastrophe of widespread triazole resistance, further understanding of the extent of crossresistance is an urgent necessity. ${ }^{2,8}$

Isavuconazole is well tolerated, as are the other azoles. In Phase I/II studies, no serious side effects were detected. The most frequent side effects were mild upper abdominal pain, moderate conjunctivitis, moderate diarrhea, moderate influenza-like illness, moderate nausea, and moderate dizziness. No visual disturbance has been reported. Until now, isavuconazole revealed no mutagenic, allergenic, phototoxic, or irritant potential. . $^{3,9,10}$

Isavuconazole has a tolerability profile somewhat similar to fluconazole. It lacks some side effects observed with voriconazole, like visual disturbance and photosensitivity.
Until now, no relevant serious adverse effects have been reported. Tolerability is an important issue for the antifungals, and in this particular case the echinocandins are considered the best example, in terms of tolerability profile.

Pharmacokinetic data on isavuconazole are available from Phase I studies. A cohort of healthy male individuals was analyzed after IV and oral administration of singleascending doses of isavuconazole. Isavuconazole doses given orally were $100 \mathrm{mg}, 200 \mathrm{mg}$, or $400 \mathrm{mg}$ equivalents of isavuconazole (corresponding to $180.5 \mathrm{mg}, 361 \mathrm{mg}$, or $722 \mathrm{mg}$ of isavuconazonium). Doses given by IV were $50 \mathrm{mg}, 100 \mathrm{mg}$, or $200 \mathrm{mg}$ equivalents of isavuconazole. The study was controlled by placebo administration. A total of 47 (23 oral and 24 IV administration) subjects were analyzed, with 33 (15 oral and $18 \mathrm{IV}$ ) of them receiving the active drug in different doses. Maximum plasma levels $\left(\mathrm{C}_{\max }\right)$ were detected 1.5-3 hours after oral administration or at the end of the IV infusion. Long half-life (56-77 hours after oral administration and 76-104 hours after IV administration) and large volumes of distribution were observed. Increases in isavuconazonium doses resulted in area under the concentrate-time curve (AUC) values increasing slightly 
more than proportionally to the dose, indicating a moderate deviation from dose linearity. Total systemic clearance (CLS/F) was low (1.91-2.80 L/h). Urinary excretion of isavuconazole was very low, ranging from $0.06 \%-0.38 \%$ when administrated via IV to $0.02 \%-0.04 \%$ when administrated orally. The similarity of AUC and $\mathrm{C}_{\max }$ parameters between oral administration and IV infusion opens the possibility of a dose interchange when switching treatments from IV to an oral route, based on the excellent bioavailability of the drug. Characteristics like low clearance, long half-life, and a large volume distribution make the drug attractive when considering alternatives for the treatment of systemic mycoses like aspergillosis or candidiasis. ${ }^{10}$

Another study assessed the effect of loading doses of isavuconazole. Healthy male subjects were randomly assigned to four treatment cohorts to receive multiple oral doses or multiple 1-hour constant-rate IV infusions of isavuconazonium. Based on the pharmacokinetic data obtained in the single-ascendingdose study mentioned previously, a considerable drug accumulation in plasma was to be expected during multiple once-daily dosing. Loading doses of isavuconazonium were equivalent to $100 \mathrm{mg}$ and $200 \mathrm{mg}$ of isavuconazole, followed by once-daily maintenance doses of $50 \mathrm{mg}$ and $100 \mathrm{mg}$, respectively, forming four cohorts of treatment. Thirty-two subjects were recruited and designated to the cohorts of treatment or placebo (two placebos per cohort). Therefore, 24 subjects received the active drug and had measured pharmacokinetics and safety parameters. One patient discontinued the study medication on study day 10 for incident illness not related to the study medication. There were no serious adverse effects. A transient elevation in liver function tests was observed in one subject with oral high-dose isavuconazole. Systemic clearance (CLSS/F) was low (2.4-2.5 L/h), and the volume of distribution (VSS/F) was large (310-350 L), as seen in the single-dose study. This study also confirmed the excellent bioavailability of isavuconazole, and intersubject variability of $\mathrm{C}_{\max }$ and AUC (0-24 hours) was low to moderate (10\%-42\%). Plasma concentrations of isavuconazole increased rapidly after administration of isavuconazonium, reaching a maximum 2.0-3.5 hours after drug intake and 0.7-1.0 hours after the IV infusion. Doses of $100 \mathrm{mg}$ daily resulted in plasma levels of $>1.7 \mu \mathrm{g} / \mathrm{mL}$ for the entire dosing interval, a concentration higher than mean fungicidal concentration (MFC) for most of the Aspergillus isolates tested. Monte Carlo simulations with Candida spp. isolates susceptible to isavuconazole (MIC of $0.06 \mu \mathrm{g} / \mathrm{mL}$ ) showed that isavuconazole is likely to exceed target AUCs sufficient to ensure clinical efficacy for patients treated with the drug. ${ }^{11,12}$
As with the other agents in the triazole class, isavuconazole can interfere in the pharmacokinetics of substrates and inhibitors of CYP3A4 isoenzyme, like rifampin, rifabutin, efavirenz, ritonavir, carbamazepine, long-acting barbiturates, phenytoin, St John's wort, and oral contraceptives. ${ }^{3,9}$ Exposure to tacrolimus was increased when coadministered with isavuconazole; administration with rifampin, a potent CYP3A4 inducer, resulted in a 35-fold increase in systemic clearance of isavuconazole, with an associated four- and 40-fold decrease in $\mathrm{C}_{\text {max }}$ and AUC. ${ }^{13}$ However, isavuconazole had no significant effect on exposure of both warfarin, a substrate of CYP2C9, ${ }^{14}$ and cyclosporine, a CYP3A4 substrate. ${ }^{15}$ Isavuconazole seems to cause less drug interaction than itraconazole or voriconazole, and probably has a profile similar to posaconazole, but data are still limited. ${ }^{3}$

An important concern with the triazole class of antifungals is its hepatic metabolism, resulting in interactions and toxicity, and limitations in patients with hepatic dysfunction. Unfortunately, this is also true with isavuconazole. A study with 48 subjects ( 16 healthy individuals, 16 with mild hepatic insufficiency, and 16 with moderate hepatic insufficiency) showed that the half-life of isavuconazole increased from 123 hours in the healthy group to 224 hours and 302 hours for subjects with mild and moderate liver impairment, respectively. Systemic clearance of isavuconazole following IV administration decreased significantly from $2.73 \mathrm{~L} / \mathrm{h}$ for healthy subjects to $1.43 \mathrm{~L} / \mathrm{h}$ for subjects with moderate liver impairment. The regimen currently used in clinical investigations (200 mg administered three times daily for the first 2 days followed by daily maintenance doses of $200 \mathrm{mg}$ ) is expected to result in substantial accumulation for patients with mild or moderate liver disease, according to the simulations made from data from this study. Therefore, it is reasonable that a dose adjustment may be needed using isavuconazole in patients with liver disease. The stronger recommendations at the moment indicate that a $50 \%$ decrease in the maintenance dose (using the same loading dose) is necessary in patients with mild and moderate hepatic disease, and the use of isavuconazole in patients with severe hepatic disease probably should be avoided. ${ }^{16}$

A study investigated 26 healthy male subjects receiving $400 \mathrm{mg}$ isavuconazole orally, as isavuconazonium, in the fed or fasted state. No significant food effect was observed. In the presence of food, the rate of absorption was delayed, but its extent was unaffected; this effect is clinically irrelevant, different from other azoles. ${ }^{17}$

The studies on the pharmacokinetics/pharmacodynamics of isavuconazole demonstrated that the bioavailability 
of this drug is very high. It has a long half-life and a large volume of distribution, a low clearance, and almost exclusively hepatic metabolism. The latter may be important regarding dosing of patients with hepatic insufficiency. High bioavailability is a common characteristic of the azole class. Unfortunately, as other members of the class, isavuconazole has significant interactions with drugs metabolized by the cytochrome P450 system, particularly isoenzyme CYP3A4. The absence of a food effect is an advantage of the drug to posaconazole and itraconazole. These advantages and disadvantages are summarized in Table 1, a comparison of pharmacokinetic and pharmacodynamic properties of isavuconazole and other azole antifungals.

\section{Could isavuconazole be of valuable use in Candida infections?}

An extensive review has been published about the in vitro activity of isavuconazole against several fungi. ${ }^{18}$ The $\mathrm{MIC}_{50}$ and $\mathrm{MIC}_{90}$ results of isavuconazole against Candida spp. are summarized in Table 2.

The in vitro activity of isavuconazole compared with fluconazole, itraconazole, voriconazole, amphotericin B, and flucytosine was evaluated against 296 clinical isolates of Candida spp. from bloodstream infections. Isavuconazole showed good activity against all Candida species, including those that were inherently less susceptible to fluconazole (eg, C. glabrata and C. krusei). Considering the $\mathrm{MIC}_{90}$, isavuconazole was as active as amphotericin $\mathrm{B}$, itraconazole, and voriconazole (each at $0.5 \mu \mathrm{g} / \mathrm{mL}$ ), and more active than flucytosine $(2 \mu \mathrm{g} / \mathrm{mL})$ and fluconazole $(8 \mu \mathrm{g} / \mathrm{mL})$. Isavuconazole has therefore demonstrated powerful in vitro activity against Candida species. ${ }^{19}$

Other studies have also shown in vitro activity of isavuconazole against most of the Candida species, with potency similar to voriconazole and posaconazole and superior to that of fluconazole, itraconazole, and amphotericin B. ${ }^{11,20-23}$ However, higher MICs of isavuconazole $(>2 \mu \mathrm{g} / \mathrm{mL})$ were observed in $C$. albicans isolates that demonstrated fluconazole MICs of $>64 \mu \mathrm{g} / \mathrm{mL},{ }^{24}$ suggesting the occurrence of cross-resistance. Against C. glabrata, C. krusei, and C. tropicalis, three of the non- $C$. albicans species that may be fluconazole resistant or require higher doses of fluconazole for treatment, the isavuconazole $\mathrm{MIC}_{50}$ values remained low, ranging between $0.25-2 \mu \mathrm{g} / \mathrm{mL}$ (C. glabrata), 0.125-0.5 $\mu \mathrm{g}$ / $\mathrm{mL}$ (C. krusei), and $0.015-0.03 \mu \mathrm{g} / \mathrm{mL}$ (C. tropicalis). Although a small number of $C$. dubliniensis isolates were evaluated, low MICs of isavuconazole have been reported in this Candida species. With other Candida species like C. lusitaniae, which may be resistant to amphotericin B, and $C$. parapsilosis and C. guilliermondii, which can present elevated MICs for the echinocandins, isavuconazole exhibited MICs that were lower than for the other antifungals tested. The MFC of isavuconazole was determined against very few Candida isolates, and no comparative data are currently available. MFC ranges varied widely $(0.03 \mu \mathrm{g} / \mathrm{mL}$ to $>16 \mu \mathrm{g} / \mathrm{mL})$ for all Candida species; however, $\mathrm{MFC}_{50}$ of $4 \mu \mathrm{g} / \mathrm{mL}$ was seen in $C$. parapsilosis, whereas $C$. albicans, C. glabrata, and C. tropicalis all exhibited $\mathrm{MFC}_{50}$ of $\geq 16 \mu \mathrm{g} / \mathrm{mL} .{ }^{18,20}$

Table I Pharmacokinetic and pharmacodynamic properties of isavuconazole compared with other azole antifungals $\mathbf{s}^{10,12,48-52}$

\begin{tabular}{|c|c|c|c|c|c|}
\hline \multirow[t]{2}{*}{ Characteristic } & \multicolumn{5}{|l|}{ Antifungal drug } \\
\hline & Isavuconazole & Voriconazole & Itraconazole & Posaconazole & Fluconazole \\
\hline Available formulations & Oral and IV & Oral and IV & Oral* & Oral & Oral and IV \\
\hline Bioavailability & Very high & Up to $95 \%$ & $\begin{array}{l}30 \% \text { capsules } \\
50 \% \text { solution }\end{array}$ & Not applicable & $95 \%$ \\
\hline Protein binding & $98 \%$ & $58 \%$ & $>99 \%$ & $99 \%$ & $10 \%$ \\
\hline Food effect & No effect & Negative effect & $\begin{array}{l}\text { Positive effect for capsules } \\
\text { Negative effect for solution }\end{array}$ & Positive effect & Negative effect \\
\hline Volume of distribution $(\mathrm{L} / \mathrm{kg})$ & High (4.4-7.7) & High (4.6) & Very high (I0.7) & High (6.5) & Low $(0.7)$ \\
\hline CNS penetration & $\begin{array}{l}\text { Low in CSF, high } \\
\text { in brain }\end{array}$ & High (>50\%) & Low $(<10 \%)$ & Low & High (>60\%) \\
\hline Clearance (L/h) & Low (1.9-2.8) & High (8.4) & Very high (I5.9) & Very high (2I.7) & Low (I.2) \\
\hline Half-life (h) & $56-104$ & $6-12$ & $24-30$ & $16-35$ & $24-30$ \\
\hline Probability of drug interactions & Moderate & High & High & Moderate & Moderate \\
\hline Use in hepatic insufficiency & $\begin{array}{l}\text { Reduce dose; } \\
\text { avoid in severe } \\
\text { hepatic disease }\end{array}$ & $\begin{array}{l}\text { Reduce dose; } \\
\text { avoid in severe } \\
\text { hepatic disease }\end{array}$ & $\begin{array}{l}\text { Reduce dose; avoid } \\
\text { in severe hepatic } \\
\text { disease }\end{array}$ & $\begin{array}{l}\text { No dose adjustment; } \\
\text { monitor carefully; avoid } \\
\text { in severe hepatic disease }\end{array}$ & $\begin{array}{l}\text { Reduce dose; avoid } \\
\text { in severe hepatic } \\
\text { disease }\end{array}$ \\
\hline
\end{tabular}

Note: *The IV formulation of itraconazole is no longer widely available in the international market.

Abbreviations: CNS, central nervous system; CSF, cerebrospinal fluid; IV, intravenous; L/h, liter/hour; h, hour. 
Table 2 Minimum inhibitory concentrations for isavuconazole, for $50 \%$ and $90 \%$ (MIC $_{50}$ and $\mathrm{MIC}_{90}$, respectively) of Candida spp. isolates. $^{18}$

\begin{tabular}{llll}
\hline Pathogen & $\begin{array}{l}\text { Antifungal } \\
\text { agent }\end{array}$ & $\begin{array}{l}\mathbf{M I C}_{50} \\
(\mu \mathrm{g} / \mathrm{mL})\end{array}$ & $\begin{array}{l}\mathbf{M I C}_{90} \\
(\mu \mathrm{g} / \mathrm{mL})\end{array}$ \\
\hline Candida albicans & Isavuconazole & $<0.002-0.03$ & $<0.002-0.03$ \\
& Voriconazole & $<0.016-0.03$ & $0.03-0.06$ \\
& Caspofungin & $0.03-0.5$ & $0.03-1$ \\
& Amphotericin B & $0.25-0.5$ & $0.5-1$ \\
Candida glabrata & Isavuconazole & $0.25-2$ & $0.5-8$ \\
& Voriconazole & $0.125-1$ & $\mathrm{I}-4$ \\
& Caspofungin & 0.125 & $0.125-0.25$ \\
& Amphotericin B & $0.5-2$ & $0.5-2$ \\
Candida krusei & Isavuconazole & $0.125-0.5$ & $0.25-1$ \\
& Voriconazole & $0.25-0.5$ & $1-4$ \\
& Caspofungin & 0.25 & $0.25-0.5$ \\
& Amphotericin B & $0.5-2$ & $0.5-4$ \\
Candida parapsilosis & Isavuconazole & $<0.015-0.03$ & $0.03-0.125$ \\
& Voriconazole & $<0.015-0.6$ & $0.06-0.125$ \\
& Caspofungin & 0.25 & $1-2$ \\
& Amphotericin B & $0.25-1$ & $0.5-2$ \\
Candida tropicalis & Isavuconazole & $<0.015-0.03$ & $0.03-0.125$ \\
& Voriconazole & $0.03-0.125$ & $0.06-2$ \\
& Caspofungin & $0.125-0.25$ & 0.5 \\
& Amphotericin B & $0.5-2$ & $0.5-2$ \\
\hline
\end{tabular}

Note: Copyright $\odot 2010$, Springer Science and Business Media. Adapted with permission from Thompson GR III, Wiederhold NP. Isavuconazole: a comprehensive review of spectrum of activity of a new triazole. Mycopathologia. 2010;170(5):29I-313.18

Abbreviation: MIC, minimum inhibitory concentration.

The efficacy of isavuconazole has been investigated in experimental models of disseminated candidiasis, ${ }^{25} \mathrm{~A}$ murine model with cyclophosphamide-induced immunosuppression was used to assess the dose response of isavuconazole along with other azoles (voriconazole and fluconazole) in C. krusei and $C$. tropicalis infections. Temporary and persistent neutropenia models were analyzed. Isavuconazole demonstrated impressive antifungal activity in both models. Against $C$. krusei, isavuconazole was superior to voriconazole (doses of $<40 \mathrm{mg} / \mathrm{kg}$ ) and fluconazole, even when administered at $150 \mathrm{mg} / \mathrm{kg}$. Isavuconazole caused a dose-dependent decline in kidney burden of C. krusei and C. tropicalis in the models. Another important finding of this animal model study was the efficacy of isavuconazole at reducing the burden of C. krusei in the brain; all doses tested significantly reduced the burden in neutropenic mice, different from other azoles. Voriconazole at $40 \mathrm{mg} / \mathrm{kg}$ was required in order to have the maximal effect in the brain. This evidence is very important because Candida central nervous system infections are a challenge to the clinician, particularly those caused by C. krusei, due to the limited therapeutic options. An agent like isavuconazole, with good tissue penetration, can be preferable to an echinocandin for invasive candidiasis in sanctuary body sites. ${ }^{25}$
Data on the pharmacokinetics and pharmacodynamics of isavuconazole have been studied in a murine model of disseminated candidiasis. Greater exposures to the drug have been demonstrated in the kidneys, with the mathematical model showing levels 5.77 times higher than those in plasma, and persistence of the drug at this site despite concentrations in plasma falling to undetectable levels. The most important parameter linked to antifungal effect was the AUC/MIC ratio. AUC/MIC ratios of 270 and 670 were associated with a $90 \%$ probability of survival in temporarily and persistently neutropenic mice, respectively. These values are similar to other azoles. ${ }^{26}$

Clinical information on isavuconazole is still limited, and data available were published only in the form of abstracts in conference proceedings. A Phase II study by Viljoen et a ${ }^{27}$ compared three dosing regimens of isavuconazonium (equivalent to $50 \mathrm{mg}$ /day and $100 \mathrm{mg} /$ day and $400 \mathrm{mg} / \mathrm{week}$ of isavuconazole) with fluconazole $100 \mathrm{mg} /$ day for treatment of Candida esophagitis. A total of 160 immunocompromised patients were recruited for the study and received treatment for 14-21 days. Endoscopically confirmed cure was achieved for $95 \%-98 \%$ of patients treated with isavuconazole (every three regimens), compared with $95 \%$ of patients in the fluconazole arm (difference not significant), therefore confirming the noninferiority of all isavuconazole regimens.

Another trial called ACTIVE, still recruiting participants, this one still recruiting participants, is a study that evaluates the role of isavuconazole in the treatment of candidemia and other invasive Candida infections (NCT00413218). It is a double-blind randomized study comparing isavuconazole versus a sequential treatment of caspofungin and voriconazole. The primary outcome measure is overall response (clinical response plus confirmed or presumed mycological response), and secondary outcome measures are overall, mycological, and clinical responses, as well as mortality and time to negative cultures. Only patients with a positive blood or a deep tissue culture positive for Candida were included. ${ }^{28}$

Another trial (NCT00413439) studied the efficacy, tolerability, and pharmacokinetics of isavuconazole in prophylaxis of IFD in patients undergoing chemotherapy for acute myeloid leukemia, and has already completed recruitment of participants. Primary outcome was safety; secondary outcomes were efficacy (frequency of invasive mycoses) and pharmacokinetic parameters. Results of this trial are not yet available. ${ }^{29}$

Isavuconazole is therefore a promising alternative for the prophylaxis and treatment of Candida infections. This assumption is based on its good in vitro activity against 
Candida species, and a significant antifungal effect in compartments like the central nervous system, as demonstrated in animal models. However, the drug still has a long way to go, due to the current lack of clinical data in patients with candidemia and the absence of comparative trials with the echinocandins. It will probably be very difficult for isavuconazole to beat the excellent safety profile and virtual lack of drug interactions of the echinocandins. Isavuconazole could, however, be a good alternative for the prophylaxis of invasive Candida infections, or maybe the drug of choice for infections caused by echinocandinresistant Candida isolates. Again, we still have to learn from the clinical trials.

\section{What is the potential role of isavuconazole in aspergillosis?}

Isavuconazole has shown potent in vitro activity against most Aspergillus species. As reviewed elsewhere, $\mathrm{MIC}_{50}$ and $\mathrm{MIC}_{90}$ results of isavuconazole against Aspergilli are described in Table $3 .{ }^{18}$ An analysis of 118 Aspergillus isolates of clinical origin, including 62 isolates of $A$. fumigatus, $20 \mathrm{~A}$. flavus, $18 \mathrm{~A}$. niger, and $18 \mathrm{~A}$. terreus, showed that isavuconazole had potent in vitro antifungal activity against all four Aspergillus species studied, including strains that were resistant to itraconazole, caspofungin, or amphotericin B. This study also demonstrated that isavuconazole had a primary fungicidal activity (presenting

Table 3 Minimum inhibitory concentrations of isavuconazole and selected antifungal agents for $50 \%$ and $90 \%\left(\mathrm{MIC}_{50}\right.$ and $\mathrm{MIC}_{90}$, respectively) of Aspergillus spp. isolates. ${ }^{18}$

\begin{tabular}{llll}
\hline Pathogen & $\begin{array}{l}\text { Antifungal } \\
\text { agent }\end{array}$ & $\begin{array}{l}\mathbf{M I C}_{50} \\
(\mu \mathrm{g} / \mathrm{mL})\end{array}$ & $\begin{array}{l}\mathrm{MIC}_{90} \\
(\mu \mathrm{g} / \mathrm{mL})\end{array}$ \\
\hline Aspergillus fumigatus & Isavuconazole & $0.25-\mathrm{I}$ & $0.5-2$ \\
& Voriconazole & $0.25-1$ & $0.5-2$ \\
& Caspofungin & $0.06-0.5$ & $0.06-0.5$ \\
& Amphotericin B & $0.25-4$ & $0.5-8$ \\
Aspergillus terreus & Isavuconazole & $0.5-1$ & $0.5-4$ \\
& Voriconazole & $0.25-1$ & $0.5-2$ \\
& Caspofungin & $0.03-0.5$ & $0.06-0.5$ \\
Aspergillus flavus & Amphotericin B & $\mathrm{I}-4$ & $\mathrm{I}-8$ \\
& Isavuconazole & $0.5-2$ & $\mathrm{I}-16$ \\
& Voriconazole & $0.5-1$ & $0.5-2$ \\
Aspergillus niger & Caspofungin & $0.03-0.25$ & $0.03-0.25$ \\
& Amphotericin B & $\mathrm{I}-2$ & $\mathrm{I}-4$ \\
& Isavuconazole & $0.5-2$ & $2-4$ \\
& Voriconazole & $0.25-2$ & $\mathrm{I}-2$ \\
& Caspofungin & $0.06-0.25$ & $0.125-0.25$ \\
& Amphotericin B & $0.5-\mathrm{I}$ & $0.5-2$ \\
\hline
\end{tabular}

Note: Copyright (C) 2010, Springer Science and Business Media. Adapted with permission from Thompson GR III, Wiederhold NP. Isavuconazole: a comprehensive review of spectrum of activity of a new triazole. Mycopathologia. 2010;170(5):29|-313.18 Abbreviation: MIC, minimum inhibitory concentration.
MFC within two dilutions of the MIC) against all four Aspergillus species. For $63 \%$ of the isolates the MFC was within one dilution of the MIC, a profile that was superior to itraconazole and voriconazole. In respect of MFCs, isolates of $A$. terreus were significantly less susceptible to isavuconazole in comparison with A. fumigatus, A. flavus, and $A$. niger. To further evaluate potential isavuconazole resistance, isolates known to have elevated posaconazole MICs were evaluated against isavuconazole. Geometric mean MIC and MFC of these isolates were $1.1 \mu \mathrm{g} / \mathrm{mL}$ and $2.3 \mu \mathrm{g} / \mathrm{mL}$, respectively. Although these values are approximately two-fold higher than the geometric mean for the total of isolates, they were within the therapeutic range of the drug, ${ }^{30}$ suggesting that isavuconazole may still retain activity against Aspergillus isolates demonstrating azole resistance.

Another study investigated the susceptibility of A. flavus to isavuconazole in comparison with other antifungal agents. A total of 208 clinical and environmental isolates were analyzed. In this study, the in vitro susceptibility to isavuconazole of 208 A. flavus isolates was determined by the European Committee on Antimicrobial Susceptibility Testing (EUCAST) method. Except for amphotericin B, all the antifungal drugs tested exhibited good in vitro activity against the majority of the $A$. flavus isolates, including isavuconazole, which had better activity compared with voriconazole. Results of isavuconazole susceptibility testing performed with the EUCAST method correlated well with those obtained by the Clinical and Laboratory Standards Institute (CLSI) method. ${ }^{31,32}$ Additionally, isavuconazole showed antifungal activity against $A$. terreus, a well-known amphotericin B-resistant Aspergillus species, with $\mathrm{MIC}_{50}$ values of below $1 \mu \mathrm{g} / \mathrm{mL}$. Against $A$. niger, isavuconazole MICs tended to be higher (one to two dilutions). ${ }^{20}$

Isavuconazole was studied in a temporarily cyclophosphamide-induced immunocompromised mouse model of disseminated $A$. flavus infection. The activity of isavuconazole was compared with other agents (voriconazole, itraconazole, and caspofungin), and outcome variables were animal survival and fungal tissue burden. Isavuconazole demonstrated great antifungal activity against $A$. flavus, leading to a prolonged survival, at least equivalent to similar doses of itraconazole and voriconazole and superior to either drug when administered at $10 \mathrm{mg} / \mathrm{kg} /$ dose. Regarding fungal burden, isavuconazole was at least as effective as itraconazole and voriconazole in reducing organ burden in all models, despite the occurrence of a lower exposure, as demonstrated by a 
four-fold lower AUC in comparison with the other drugs. In this study, tissue burden was assessed by both conventional culture and quantitative real-time polymerase chain reaction using fluorescence resonance energy transfer technology, a more accurate method to determine the organ burden in filamentous fungus infections, in comparison with conventional quantitative fungal culture. ${ }^{5}$

Another clinical trial (NCT00412893) with isavuconazole has already completed enrollment. This was a double-blind, randomized study called SECURE, that aimed to compare the efficacy and safety of isavuconazole versus voriconazole in the primary treatment of IFDs caused by Aspergillus or other filamentous fungi. Primary outcome measure was mortality through day 42 after treatment, and secondary outcome measures were clinical, mycological, and radiological response. The inclusion criterion was the diagnosis of probable or proven IFD caused by Aspergillus species or other filamentous fungi. Patients with other invasive fungal infections (neither Aspergillus nor filamentous fungi), chronic aspergillosis (either aspergilloma or allergic bronchopulmonary aspergillosis), previous systemic antifungal therapy other than fluconazole, or body weight under $40 \mathrm{~kg}$ were excluded from the study. ${ }^{33}$ Astellas Pharma has recently reported, in a press release, important data from this study. Isavuconazole was effective in the treatment of IA as determined by the primary endpoint of all-cause mortality through day 42 in the intent-to-treat population $(n=516)$. The all-cause-mortality was $18.6 \%$ in the isavuconazole treatment group and $20.2 \%$ in the voriconazole group; isavuconazole had achieved noninferiority criteria. Secondary endpoint of overall success rate was also similar between isavuconazole and voriconazole arms. No differences in adverse events were observed as well. Although not yet published in scientific grounds, these preliminary results indicate that isavuconazole will probably have an important role in the treatment of IA. ${ }^{34}$

The latest trial is an evaluation of isavuconazole in the treatment of aspergillosis and other rare fungi infections in patients with renal impairment (NCT00634049). This trial called VITAL, has recently completed enrollment, and it is an open-label interventional study. Primary outcome measure is overall response (clinical, mycological, and radiological) determined by an external committee, and secondary measures are overall response determined by the investigator, clinical and mycological responses, and survival rate. Patients with probable or proven IFD caused by Aspergillus species with renal impairment or by rare molds, yeasts, or dimorphic fungi with or without renal impairment were allowed to be included. Patients with a probable or proven mucormycosis (whether renally impaired or not) could also be included. ${ }^{35}$ One hundred and fifty patients were included. Review of diagnosis and outcomes by an Independent Data Review Committee is ongoing. ${ }^{34}$

Isavuconazole therefore has several attractive features that may put this agent as a potential first-line drug to treat IA. Many studies have demonstrated that isavuconazole has potent in vitro activity against the Aspergilli, which has gained additional support in studies utilizing animal models of aspergillosis. Isavuconazole also has pharmacological advantages in comparison with voriconazole, including the absence of cyclodextrin in its IV formulations, possibility of once-a-day dosing, and probably a better tolerability profile. Preliminary results reported have demonstrated the potential of isavuconazole in the treatment of IA. Nevertheless, results of the Phase III trials need to be known in detail before any further assumption can be made on the role of isavuconazole in the treatment of patients with IA.

\section{Isavuconazole: a new hope for mucormycosis treatment?}

Mucormycosis is a severe disease in human hosts, and the causative organisms usually respond poorly to antifungal therapy. Amphotericin B formulations remain the mainstay of therapy against mucormycosis. The in vitro activity of posaconazole against these fungi has been well demonstrated elsewhere, ${ }^{36-38}$ and its efficacy in clinical settings has thus far been studied only in salvage therapy. ${ }^{39}$

Regarding the Mucorales, frequent agents of mucormycosis, a study evaluated the in vitro activity of isavuconazole against a collection of 345 diverse Mucorales isolates, collected and tested at eight study centers in Europe, Mexico, and North America. In vitro susceptibility testing of the isolates was determined according to EUCAST or CLSI guidelines. Isavuconazole exhibited $\mathrm{MIC}_{50}$ values of $1-4 \mu \mathrm{g} /$ $\mathrm{mL}$ and $\mathrm{MIC}_{90}$ values of $4-16 \mu \mathrm{g} / \mathrm{mL}$, against the five fungal genera, although there were also marked differences in MIC distributions. EUCAST methods appeared to generate modal MICs two-fold higher than CLSI. The results confirmed the at least partial antifungal activity of isavuconazole against the Mucorales. ${ }^{40}$

In a study comparing the in vitro activity of posaconazole against the agents of mucormycosis, isavuconazole showed comparable $\mathrm{MIC}_{90}$ values, although readings were usually two- to four-fold higher. Isavuconazole $\mathrm{MIC}_{50}$ values were lowest in Lichtheimia (formerly Absidia) $(0.5-4 \mu \mathrm{g} / \mathrm{mL})$ and Rhizopus spp. (0.25-4 $\mu \mathrm{g} / \mathrm{mL})$ compared with other species. 
However, posaconazole presented lower MICs $(0.12-1 \mu \mathrm{g} / \mathrm{mL}$ and $0.25-2 \mu \mathrm{g} / \mathrm{mL}$, respectively). Isavuconazole exhibited higher $\mathrm{MIC}_{90}$ values in comparison with amphotericin B for all fungal species evaluated in the study. MFC values of isavuconazole were typically high against these organisms. ${ }^{18}$

The activity of isavuconazole against the agents of mucormycosis is a positive characteristic of this drug and a potential advantage in terms of antifungal spectrum in comparison with the other azoles. Considering that isavuconazole is also available via IV, different from posaconazole, this favors isavuconazole as a preferential agent for use in combination regimens with other antifungals such as the polyenes. In the VITAL trial, approximately 45 patients were enrolled with mucormycosis; results have not yet been reported. Clinical data will dictate whether isavuconazole has any role in the treatment of mucormycosis, considering that isavuconazole is apparently less active than amphotericin B and posaconazole against the agents of mucormycosis. ${ }^{34,35}$

\section{Cryptococcus and other emerging yeast infections: does isavuconazole have the potential for therapy?}

Isavuconazole has potent activity against Cryptococcus spp., and it has demonstrated superior activity against these fungi in comparison with the currently licensed antifungal drugs. A case series from Cuba involving 165 clinical and environmental isolates of Cryptococcus spp. revealed that isavuconazole had low MICs against these isolates. The study involved mostly Cryptococcus neoformans variety grubii, except for one obtained from an animal (Cryptococcus gattii) and two obtained from environmental samples from Pinar del Rio (Cryptococcus albidus and Cryptococcus albidisimilus). Another study evaluating the in vitro activity of isavuconazole against Cryptococcus included a total of 128 isolates; of these, 86 were $C$. neoformans (28 of serotype A, 25 of serotype D, and 33 of the hybrid AD serotype) and 42 were $C$. gattii (30 of serotype B and twelve of serotype C). Samples were collected from both clinical and environmental sources, from the United States, Australia, France, Denmark, Italy, Thailand, and the Democratic Republic of Congo. Isavuconazole, posaconazole, and voriconazole demonstrated excellent in vitro activity against all Cryptococcus serotypes tested. ${ }^{41-43}$

Isavuconazole has potent in vitro activity against the emerging yeasts. Trichosporon isolates had isavuconazole $\mathrm{MIC}_{90}$ values lower than those of amphotericin B and flucytosine, and lower than, or equivalent to, the corresponding values of other azoles, except for voriconazole, which presented MIC values that were two- to four-fold lower. ${ }^{44}$ Similar results were seen in the other rare yeasts that were tested in the study, such as Geotrichum capitatum and Pichia spp. Against these fungal genera, isavuconazole, voriconazole, and posaconazole had similar activity, with $\mathrm{MIC}_{90}$ values ranging from $0.03 \mu \mathrm{g} / \mathrm{mL}$ to $0.5 \mu \mathrm{g} / \mathrm{mL}$. Against species of Rhodotorula and Saccharomyces, isavuconazole, along with voriconazole, was found to be one of the most potent antifungal agents in the study. ${ }^{18,44}$

Isavuconazole will probably have a role in the treatment of cryptococcosis as an alternative to the current first-line drugs. Isavuconazole penetrates well in the central nervous system, as demonstrated in animal models (against Candida). In induction therapy, isavuconazole would hardly overcome the fungicidal effect and optimal central nervous system penetration of amphotericin $\mathrm{B}$, but its favorable safety profile could be sufficient to result in a better clinical outcome. For maintenance therapy, upfront it has no clear advantage over fluconazole, another azole agent with excellent tolerability and safety, and also available via IV. Again, clinical data are definitely needed to answer these questions.

\section{What is the role of isavuconazole against other fungal infections, including fusariosis and phaeohyphomycosis?}

Other medically important molds like Fusarium and Scedosporium spp. have demonstrated reduced susceptibility to most antifungal agents. Isavuconazole was found to be active against Fusarium species, with low MICs. However, the $\mathrm{MIC}_{50}$ values were equivalent to, or higher than, the other azoles, like posaconazole and voriconazole, and to echinocandins. ${ }^{18}$

Isavuconazole $\mathrm{MIC}_{50}$ against $S$. apiospermum was similar to the extended spectrum triazoles (lower values for voriconazole and posaconazole, in comparison with isavuconazole). Against $S$. prolificans, all of the evaluated azoles exhibited high MIC values ( $>4 \mu \mathrm{g} / \mathrm{mL})$. Additionally, isavuconazole MFCs were very high, exceeding $16 \mu \mathrm{g} / \mathrm{mL}$ against this species. Isavuconazole has shown high activity against Paecilomyces spp., with low MICs $(1 \mu \mathrm{g} / \mathrm{mL})$, lower than, or equal to, those of all other tested antifungal agents. ${ }^{18}$

Against dematiaceous fungi (including the species Alternaria, Bipolaris, Cladosporium, Curvularia, Exophiala, Fonsecaea, and Phialophora), very low MICs to isavuconazole were observed. The extended spectrum triazoles, including isavuconazole, have shown lower $\mathrm{MIC}_{50}$ against Alternaria alternata, in comparison with Alternaria 
infectoria. ${ }^{18}$ Isavuconazole $\mathrm{MIC}_{50}$ values were less than, or equivalent to, other triazoles against $B$. spicifera, $C$. lunata, Exophiala spp., and Rhinocladiella mackenziei. ${ }^{18,45}$ For Cladophialophora spp., isavuconazole MICs were at least three dilutions lower than those of amphotericin B. ${ }^{18}$

The in vitro activity of isavuconazole against Madurella mycetomatis was evaluated by determining the MICs of 22 M. mycetomatis strains from Sudan. Compared with ketoconazole, significantly lower MICs were seen for isavuconazole, ranging from $0.016 \mu \mathrm{g} / \mathrm{mL}$ to $0.125 \mu \mathrm{g} / \mathrm{mL}$, with an $\mathrm{MIC}_{90}$ of $0.063 \mu \mathrm{g} / \mathrm{mL}$. Compared with other studies that determined MICs of posaconazole and voriconazole, MICs of isavuconazole were significantly lower than those of voriconazole, but not those of posaconazole. The authors concluded that isavuconazole seems to be highly active against $M$. mycetomatis, with lower MICs than ketoconazole. ${ }^{46}$

Isavuconazole has shown very potent in vitro activity against the dermatophytes (Trichophyton rubrum, Trichophyton mentagrophytes, Trichophyton tonsurans, Epidermophyton floccosum, and Microsporum canis), with MIC values lower than for the other triazoles. Only terbinafine demonstrated MIC values that were lower than those of isavuconazole. Against terbinafine-resistant T. rubrum strains, isavuconazole MICs remained low. ${ }^{18}$

Isavuconazole had potent in vitro activity against the dimorphic fungi. The MICs of isavuconazole were similar to all other tested agents against Blastomyces dermatitidis, Histoplasma capsulatum, and Coccidioides posadasii, and were lower than those of fluconazole. Nevertheless, against species of Sporothrix, isavuconazole demonstrated higher $\mathrm{MIC}_{90}$ values than fluconazole. ${ }^{18}$

Rare yeast pathogens have also been tested for susceptibility to isavuconazole in a study that evaluated 74 isolates (Dipodascus capitatus $[\mathrm{n}=21]$; Rhodotorula mucilaginosa $[\mathrm{n}=18]$; Saccharomyces cerevisiae $[\mathrm{n}=20]$; Trichosporon spp. [n=15]: T. mucoides $[\mathrm{n}=8]$, T. inkin $[\mathrm{n}=3]$, T. jirovecii $[\mathrm{n}=2]$, T. domesticum $[\mathrm{n}=1]$, and T. asahii $[\mathrm{n}=1])$. MICs of isavuconazole, posaconazole, voriconazole, and fluconazole were determined by broth microdilution and E-test. Isavuconazole had reasonably low MICs for most of the strains examined; it was the only azole that showed partial antifungal activity against $R$. mucilaginosa. The results corroborated that isavuconazole is likely to be effective against infections caused by D. capitatus, S. cerevisiae, Trichosporon spp., or Rhodotorula. ${ }^{47}$

Isavuconazole is probably equal to the other azoles, with rare exceptions, against Fusarium, dematiaceous fungi, Madurella, and dimorphic fungi, regarding in vitro antifungal activity. Pharmacological advantages could benefit isavuconazole as a preferable agent to be used alone or in combination in the treatment of infections by these organisms, but still no animal model or clinical data are available.

\section{Conclusion}

Here we have reviewed the drug profile, in vitro activity, pharmacokinetics and pharmacodynamics, safety, and clinical efficacy of isavuconazole, a new-generation azole. Isavuconazole has a broad spectrum of antifungal activity, being highly active against most medically important fungi.

Differently from voriconazole, isavuconazole is administrated as a prodrug that is water soluble and therefore does not need the addition of cyclodextrin to IV formulations, eliminating concerns for nephrotoxicity due to this component. Differently from posaconazole, isavuconazole is also available via IV, an important advantage in the treatment of serious fungal infections. Another difference from voriconazole and posaconazole is the apparent absence of a food effect with isavuconazole. Regarding antifungal drug resistance, data are still very limited, but it is reasonable to assume that isavuconazole will also be affected by cross-resistance that is also seen for the other new-generation azoles. The metabolism of isavuconazole is not substantially distinct from other azoles. Interactions with cytochrome P450 substrates, inducers, and inhibitors are therefore expected. Characteristics such as low clearance, long half-life, and a large volume distribution, well observed in pharmacokinetic/pharmacodynamic studies, assure the possibilities of using this drug for treatment of IFDs.

Isavuconazole thus has a great potential for the treatment of invasive fungal infections. However, there is a need for further clinical efficacy data to endorse its indication as an appropriate therapy for these serious conditions. Until now, safety seems not to have been a significant concern. Good tolerability and low incidence of adverse events have been observed. Efficacy in esophageal candidiasis has already been demonstrated. Despite promising preliminary results, reported very recently, there are no published data in other clinical conditions such as aspergillosis or other IFDs. The proper role of isavuconazole is still to be defined by the upcoming results of clinical trials.

\section{Disclosure}

The authors report no conflicts of interest in this work.

\section{References}

1. Girmenia C. New generation azole antifungals in clinical investigation. Expert Opin Investig Drugs. 2009;18(9):1279-1295. 
2. Livermore J, Hope W. Evaluation of the pharmacokinetics and clinical utility of isavuconazole for treatment of invasive fungal infections. Expert Opin Drug Metab Toxicol. 2012;8(6):759-765.

3. Pasqualotto AC, Denning DW. New and emerging treatments for fungal infections. J Antimicrob Chemother. 2008;61(Suppl 1):i19-i30.

4. Kagoshima Y, Mori M, Suzuki E, et al. Design, synthesis and antifungal activity of a novel water soluble prodrug of antifungal triazole. Bioorg Med Chem Lett. 2003;13(2):191-196.

5. Warn PA, Sharp A, Mosquera J, et al. Comparative in vivo activity of BAL4815, the active component of the prodrug BAL8557, in a neutropenic murine model of disseminated Aspergillus flavus. J Antimicrob Chemother. 2006;58(6):1198-1207.

6. Denning DW, Hope WW. Therapy for fungal diseases: opportunities and priorities. Trends Microbiol. 2010;18(5):195-204.

7. Chowdhary A, Kathuria S, Randhawa HS, Gaur SN, Klaassen CH, Meis JF. Isolation of multiple-triazole-resistant Aspergillus fumigatus strains carrying the TR/L98H mutations in the CYP51A gene in India. $J$ Antimicrob Chemother. 2012;67(2):362-366.

8. Howard SJ, Cerar D, Anderson MJ, et al. Frequency and evolution of azole resistance in Aspergillus fumigatus associated with treatment failure. Emerg Infect Dis. 2009;15(7):1068-1076.

9. Guinea J, Bouza E. Isavuconazole: a new and promising antifungal triazole for the treatment of invasive fungal infections. Future Microbiol. 2008;3(6):603-615.

10. Schmitt-Hoffmann A, Roos B, Heep M, et al. Single-ascending-dose pharmacokinetics and safety of the novel broad-spectrum antifungal triazole BAL4815 after intravenous infusions (50, 100, and 200 milligrams) and oral administrations (100, 200, and 400 milligrams) of its prodrug, BAL8557, in healthy volunteers. Antimicrob Agents Chemother. 2006;50(1):279-285.

11. Ostrosky-Zeichner L, Inurria N, Rodriguez J, Chen E, Paetznick V. Comparative in vitro activity of isavuconazole (ISA) against medically important yeasts and moulds. Paper presented at: Programs and Abstracts of the 49th Annual; Interscience Conference on Antimicrobial Agents and Chemotherapy (ICAAC); 2009; San Francisco, CA.

12. Schmitt-Hoffmann A, Roos B, Maares J, et al. Multiple-dose pharmacokinetics and safety of the new antifungal triazole BAL4815 after intravenous infusion and oral administration of its prodrug, BAL8557, in healthy volunteers. Antimicrob Agents Chemother. 2006;50(1):286-293.

13. Schmitt-Hoffmann A, Roos B, Sauer J. Effect of rifampicin on the pharmacokinetics of BAL4815 at steady state after multiple oral doses of BAL8557 (WSA) and rifampicin. Paper presented at: Abstracts of the Sixteenth Congress of the International Society for Human and Animal Mycology (ISHAM); 2006; Paris, France.

14. Schmitt-Hoffmann A, Roos B, Sauer J. Effect of BAL8557, a watersoluble azole pro-drug (WSA), on the pharmacokinetics of S- and R-warfarin. Paper presented at: Abstracts of the Sixteenth Congress of the International Society for Human and Animal Mycology (ISHAM); 2006; Paris, France.

15. Schmitt-Hoffmann A, Roos B, Sauer J. Effect of BAL8557, a water-soluble azole pro-drug (WSA), on the pharmacokinetics of ciclosporin. Paper presented at: Abstracts of the Sixteenth Congress of the International Society for Human and Animal Mycology (ISHAM); 2006; Paris, France.

16. Schmitt-Hoffmann A, Roos B, Spickermann J, et al. Effect of mild and moderate liver disease on the pharmacokinetics of isavuconazole after intravenous and oral administration of a single dose of the prodrug BAL8557. Antimicrob Agents Chemother. 2009;53(11):4885-4890.

17. Schmitt-Hoffman A, Roos B, Spickerman J, et al. No relevant food effect in man on isavuconazole oral pharmacokinetics preliminary data. Paper presented at: 48th Annual International Conference on Antimicrobial Agents and Chemotherapy (ICAAC); 2008; Washington, DC.

18. Thompson GR III, Wiederhold NP. Isavuconazole: a comprehensive review of spectrum of activity of a new triazole. Mycopathologia. 2010;170(5):291-313.

19. Seifert H, Aurbach U, Stefanik D, Cornely O. In vitro activities of isavuconazole and other antifungal agents against Candida bloodstream isolates. Antimicrob Agents Chemother. 2007;51(5):1818-1821.
20. Guinea J, Pelaez T, Recio S, Torres-Narbona M, Bouza E. In vitro antifungal activities of isavuconazole (BAL4815), voriconazole, and fluconazole against 1,007 isolates of zygomycete, Candida, Aspergillus, Fusarium, and Scedosporium species. Antimicrob Agents Chemother. 2008;52(4):1396-1400.

21. Curfs-Brekuer IM, Mouton J, Debets-Ossenkpp YJ, Endtz HP, Verweij PE, Meis JF. In vitro activity of isavuconazole (BAL4815) compared with seven other antifungal agents against 309 prospectively collected clinical Candida isolates from The Netherlands. Paper presented at: Programs and Abstracts of the 17th European Congress of Clinical Microbiology and Infectious Diseases (ECCMID); 2007; Munich, Germany.

22. Cuenca-Estrella A, Gomez-Lopez A, Buitrago MJ, Mellado E, Rodrigues-Tudela JL. BAL4815 a new antifungal agent: determination of quality control limits for susceptibility testing by methods of the european committee on antimicrobial susceptibility testing (EUCAST). Paper presented at: Programs and Abstracts of the 46th Annual International Conference on Antimicrobial Agents and Chemotherapy (ICAAC) Annual Meeting; 2006; San Francisco, CA.

23. Heep M, Grover P, Brown NP, Pillar CM, Jones ME, Sahm DF. Evaluation of isavuconazole e-test compared to broth microdilution antifungal susceptibility testing against quality control strains and clinical Candida isolates. Paper presented at: Programs and Abstracts of the 17th European Congress of Clinical Microbiology and Infectious Diseases (ECCMID); 2007; Munich, Germany.

24. Sanglard D, Ischer F, Coste A, Rerrari S. Comparison between isavuconazole (ISA) and other azoles against characterized clinical isolates and yeast model systems. Paper presented at: Abstracts of the 18th Annual European Congress of Clinical Microbiology and Infectious Diseases (ECCMID); 2008; Barcelona, Spain.

25. Majithiya J, Sharp A, Parmar A, Denning DW, Warn PA. Efficacy of isavuconazole, voriconazole and fluconazole in temporarily neutropenic murine models of disseminated Candida tropicalis and Candida krusei. J Antimicrob Chemother. 2009;63(1):161-166.

26. Warn PA, Sharp A, Parmar A, Majithiya J, Denning DW, Hope WW. Pharmacokinetics and pharmacodynamics of a novel triazole, isavuconazole: mathematical modeling, importance of tissue concentrations, and impact of immune status on antifungal effect. Antimicrob Agents Chemother. 2009;53(8):3453-3461.

27. Viljoen J, Mitha I, Heep M, Ghannoum MA. Efficacy, safety, and tolerability of three different dosing regimens of BAL8557 vs fluconazole in a double-blind, randomized, multicenter trials for the treatment of esophageal candididasis in immunocompromissed adults. Paper presented at: Abstracts of the 45th Interscience Conference on Antimicrobial Agents and Chemotherapy (ICAAC); 2005; Washington, DC.

28. National Institutes of Health. Isavuconazole (BAL8557) in the treatment of candidemia and other invasive Candida infections. In: ClinicalTrials. gov [website on the Internet]. Bethesda, MD: US National Library of Medicine; 2013. Available from: http://www.clinicaltrials.gov/ ct2/show/NCT00413218?term=00413218\&rank=1. NLM identifer: NCT00413218. Accessed August 16, 2013.

29. National Institutes of Health. Efficacy and pharmacokinetics of an antifungal in patients undergoing chemotherapy. In: ClinicalTrials.gov [website on the Internet]. Bethesda, MD: US National Library of Medicine; 2011. Available from: http:/www.clinicaltrials.gov/ct2/show/NCT00413439?term=004134 39\&rank=1. NLM identifier: NCT00413439. Accessed August 16, 2013.

30. Warn PA, Sharp A, Denning DW. In vitro activity of a new triazole BAL4815, the active component of BAL8557 (the water-soluble prodrug), against Aspergillus spp. J Antimicrob Chemother. 2006;57(1):135-138.

31. Rudramurthy SM, Chakrabarti A, Geertsen E, Mouton JW, Meis JF. In vitro activity of isavuconazole against 208 Aspergillus flavus isolates in comparison with 7 other antifungal agents: assessment according to the methodology of the European Committee on Antimicrobial Susceptibility Testing. Diagn Microbiol Infect Dis. 2011;71(4):370-377.

32. Shivaprakash MR, Geertsen E, Chakrabarti A, Mouton JW, Meis JF. In vitro susceptibility of 188 clinical and environmental isolates of Aspergillus flavus for the new triazole isavuconazole and seven other antifungal drugs. Mycoses. 2011;54(5):e583-e589. 
33. National Institutes of Health. Isavuconazole (BAL8557) for primary treatment of invasive Aspergillosis. In: ClinicalTrials.gov [website on the Internet]. Bethesda, MD: US National Library of Medicine; 2013. Available from: http://www.clinicaltrials.gov/ct2/show/NCT00412893 ?term $=00412893 \&$ rank=1. NLM identfier: NCT00412893. Accessed August 16, 2013.

34. Astellas Pharma Inc. Astellas reports positive topline phase 3 results for antifungal isavuconazole. In: www.astellas.com/en [website on the Internet]. Tokyo, Japan; 2013. Available from: http://www.astellas. com/en/corporate/news/pdf/130930_2_Eg.pdf. Accessed September $30,2013$.

35. National Institutes of Health. Isavuconazole in the treatment of renally impaired aspergillosis and rare fungi. In: ClinicalTrials.gov [website on the Internet]. Bethesda, MD: US National Library of Medicine; 2013. Available from: http:/www.clinicaltrials.gov/ct2/show/NCT0063404 9?term=isavuconazole+renal+impairment\&rank=2. NLM identifier: NCT00634049. Accessed August 16, 2013.

36. Alastruey-Izquierdo A, Castelli MV, Cuesta I, et al. In vitro activity of antifungals against Zygomycetes. Clin Microbiol Infect. 2009; 15(Suppl 5):71-76.

37. Almyroudis NG, Sutton DA, Fothergill AW, Rinaldi MG, Kusne S. In vitro susceptibilities of 217 clinical isolates of zygomycetes to conventional and new antifungal agents. Antimicrob Agents Chemother. 2007;51(7):2587-2590.

38. Sun QN, Najvar LK, Bocanegra R, Loebenberg D, Graybill JR. In vivo activity of posaconazole against Mucor spp. in an immunosuppressed-mouse model. Antimicrob Agents Chemother. 2002;46(7): 2310-2312.

39. Enoch DA, Aliyu SH, Sule O, Lewis SJ, Karas JA. Posaconazole for the treatment of mucormycosis. Int J Antimicrob Agents. 2011;38(6): 465-473.

40. Verweij PE, González GM, Wiedrhold NP, Lass-Flörl C, et al. In vitro antifungal activity of isavuconazole against 345 Mucorales isolates collected at study centers in eight countries. J Chemother. 2009;21(3): $272-281$.

41. Illnait-Zaragozi MT, Martinez GF, Curfs-Breuker I, Fernandez CM, Boekhout T, Meis JF. In Vitro activity of the new azole isavuconazole (BAL4815) compared with six other antifungal agents against 162 Cryptococcus neoformans isolates from Cuba. Antimicrob Agents Chemother. 2008;52(4):1580-1582.
42. Thompson GR III, Fothergill AW, Wiederhold NP, Vallor AC, Wickes BL, Patterson TF. Evaluation of Etest method for determining isavuconazole MICs against Cryptococcus gattii and Cryptococcus neoformans. Antimicrob Agents Chemother. 2008;52(8):2959-2961.

43. Thompson GR III, Wiederhold NP, Fothergill AW, Vallor AC, Wickes BL, Patterson TF. Antifungal susceptibilities among different serotypes of Cryptococcus gattii and Cryptococcus neoformans. Antimicrob Agents Chemother. 2009;53(1):309-311.

44. Thompson GR III, Wiederhold NP, Sutton DA, Fothergill A, Patterson TF. In vitro activity of isavuconazole against Trichosporon, Rhodotorula, Geotrichum, Saccharomyces and Pichia species. J Antimicrob Chemother. 2009;64(1):79-83.

45. Gonzalez GM. In vitro activities of isavuconazole against opportunistic filamentous and dimorphic fungi. Med Mycol. 2009;47(1):71-76.

46. Kloezen W, Meis JF, Curfs-Breuker I, Fahal AH, van de Sande WW. In vitro antifungal activity of isavuconazole against Madurella mycetomatis. Antimicrob Agents Chemother. 2012;56(11):6054-6056.

47. Guinea J, Recio S, Escribano P, Pelaez T, Gama B, Bouza E. In vitro antifungal activities of isavuconazole and comparators against rare yeast pathogens. Antimicrob Agents Chemother. 2010;54(9):4012-4015.

48. Brammer KW, Farrow PR, Faulkner JK. Pharmacokinetics and tissue penetration of fluconazole in humans. Rev Infect Dis. 1990;12 (Suppl 3): S318-S326.

49. Courtney R, Pai S, Laughlin M, Lim J, Batra V. Pharmacokinetics, safety, and tolerability of oral posaconazole administered in single and multiple doses in healthy adults. Antimicrob Agents Chemother. 2003;47(9):2788-2795.

50. Leveque D, Nivoix Y, Jehl F, Herbrecht R. Clinical pharmacokinetics of voriconazole. Int J Antimicrob Agents. 2006;27(4):274-284.

51. Moton A, Krishna G, Ma L, et al. Pharmacokinetics of a single dose of the antifungal posaconazole as oral suspension in subjects with hepatic impairment. Curr Med Res Opin. 2010;26(1):1-7.

52. Patel K, Roberts JA, Lipman J, Tett SE, Deldot ME, Kirkpatrick CM. Population pharmacokinetics of fluconazole in critically ill patients receiving continuous venovenous hemodiafiltration: using Monte Carlo simulations to predict doses for specified pharmacodynamic targets. Antimicrob Agents Chemother. 2011;55(12):5868-5873.
Infection and Drug Resistance

\section{Publish your work in this journal}

Infection and Drug Resistance is an international, peer-reviewed openaccess journal that focuses on the optimal treatment of infection (bacterial, fungal and viral) and the development and institution of preventive strategies to minimize the development and spread of resistance. The journal is specifically concerned with the epidemiology of antibiotic

\section{Dovepress}

resistance and the mechanisms of resistance development and diffusion in both hospitals and the community. The manuscript management system is completely online and includes a very quick and fair peerreview system, which is all easy to use. Visit http://www.dovepress.com/ testimonials.php to read real quotes from published authors. 